\title{
Application of Spread Spectrum Digital Train-tail Device in Shenshuo
}

\author{
Railway Line \\ Liping Wang ${ }^{1, a^{*}}$, Sanbo Yang ${ }^{2, b}$ and Fuyun $\mathrm{Zhao}^{2, \mathrm{c}}$ \\ ${ }^{1}$ Xijing University, Xi'an, Shaanxi, China \\ ${ }^{2}$ Shaanxi Fenghuo Group, Baoji, Shaanxi, China \\ awlpggg@163.com, b108766@qq.com, 65615252@qq.com \\ *The corresponding author
}

Keywords: Train-tail device; Digital spread spectrum; Communication; Railway; Safety

\begin{abstract}
Digital spread spectrum communication technology has the advantages of anti-noise, anti-jamming, anti-fading, anti-multipath and multi-address communication. In this paper, digital spread spectrum communication technology is used in the train tail safety protection device. The utility model overcomes the defects of poor anti-interference ability and single function of the existing analog tail device, to meet the 10,000 tons of freight locomotive communication requirements. It Improve the reliability of locomotive communication, to ensure the safe operation of the train.
\end{abstract}

\section{Introduction}

The train-tail device is a special equipment to ensure the safety for railway transport in unattended circumstances. Based on the wireless communication, the tail device realize communication between the locomotive station with the end of the train and communication between the different locomotive stations. It is very important to realize the safety control of the locomotive to the tail of the train and the tail information automatic feedback of the train through the remote detection of the radio signal. When the main air pressure drops to the specified threshold due to abnormal leakage, the tail device will automatically alarms. When the angle cock is closed accidentally, the crew can operate the tail device to exhaust auxiliary brake, making sure the safety of the train and preventing the train locomotive service being out of the control. The tail-to-tail device consists of a locomotive station mounted on a locomotive and a tail-mounted host attached to the rear of the train.

\section{Present Problems of the Existing Train-Tail Devices}

In order to improve the transport capacity, a train takes two sets of locomotive has improved the transport capacity to more than 10,000 tons per column transport, such as Datong Railway, which is belong to Shenhua Group. As the tons of transport mode increase the communication distance, the fading of the signal is speeded up. The new installation of a variety of electrical equipment make variety of locomotor electromagnetic interference more serious, resulting in data transmission error rate increases. As a result, the above factors make the tail device communication instable. Because the two groups of locomotives need to communicate voice signal simultaneously, it would produce the same frequency crosstalk, especially when the terrain environment is more complex and the electromagnetic environment is worse.

\section{Communication Requirements of Million Tons Transport in Shenshuo Railway Line}

The Basic Operation Condition. The railway transport line of Shenshuo railway company is from the Shenmu County North Station to the Shenchi County South Station in Shaanxi Province (referred to as Shennan). The total length of the railway is $168 \mathrm{Km}$ and Running for about 4 hours. 
Mountains and tunnels account for about $60 \%$ of the total shennan railway. There is 2 tunnels which is more than $5 \mathrm{Km}$ and the uplink (Shenbei - Shennan) for the heavy load $0.3 \%$ uphill in the railway. It is one of the more complex operating environment in the domestic railway line. Because of the complicated situation, the locomotive take a $2+2$ group combination: that is, a group of locomotives +5000 tons of train +1 group of locomotives +5000 tons of trains. (Fig. 1)

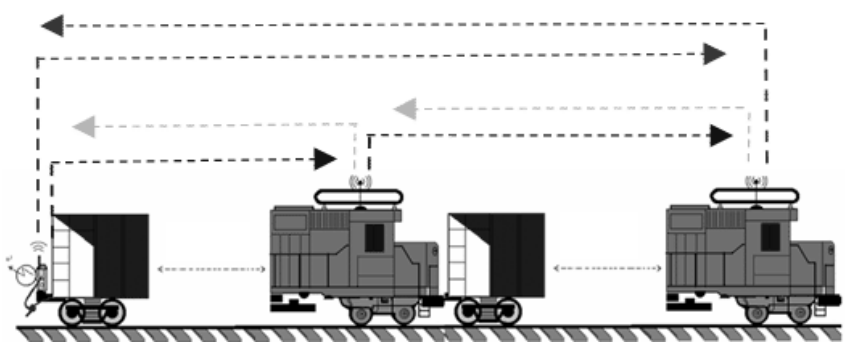

Figure 1. Million tons of locomotives and communication methods

Basic Communication Requirements. The characteristic parameter are:

a. The "one-to-one" data communication between the locomotive station and the tail device;

b. Marshalling between the locomotive stations;

c. Data communication rate is not less than $93 \%$ across the whole line; voice communication rate is not less than $98 \%$;

Basic Operating Requirements. The characteristic parameter are:

a. Eight locomotive stations are installed in the two sets of locomotives with train-tail device. There are 4 sets in uplink communication path and 4 sets in downlink communication path. The front locomotive is set as "host car", then the following is "slave car 1", "slave car 2", "slave car $3 "$, and a train-tail host is installed in the end of the train .

b. All the marshalling locomotive stations can query the data of the host and receive the data information from the train-tail host.

c. Marshalling locomotive stations communicates bettwen each other in the simplex mode.

\section{Basic Communication Scheme}

The communication mode of train-tail device is usually used in both the induction communication (which is conducive to the transmission of the locomotive power line transmission signal) and the ultra-short wave communication. According to the road environment and the communication requirements of the 10,000 tons locomotive, the digital communication technology is used in the train-tail device to improve the communication reliability and the anti-interference ability. To achieve operational requirements, digital spread spectrum communication technology are used anti-jamming performance with the extended frequency communication technology. Spread spectrum communication is more advantageous communication mode, and it is also one of the hotspots of international hi-tech. It was used mainly for military communications in early stadge, then rapidly spread in the field of civilian communications. Spread spectrum communication technology is a major breakthrough in communication technology. Software radio (SDR) is used to achieve the transmission and processing of information, which has a high degree of modularity, anti-interference and anti-signal fading ability. It can work in negative threshold level conditions to improve the complex environment adaptability and communication capacity.

Spread Spectrum Communication Scheme Using 400khz Induction Communication. The current induction communication uses the analog standard: the voice communication uses the FM mode, and the data communication uses FFSK. The information processing is realized by the hardware from the baseband to the frequency band. The squelch mode is sub-audio coding, and the data communication is received at the error rate of 10-5 with the limit of the threshold level is about $6 \mathrm{~dB}$. The launch power of the train-tail host is about $2 \mathrm{~W}$ in current circumstances, which improve the main contradiction in communication without increasing the transmission power to 
improve the receiving field strength. So, the usage of spread spectrum communication technology is an correct method to reduce the receiving threshold level. The current sensor antenna parameters are:

a. Frequency: 490KHz;

b. Spread spectrum bandwidth: $30 \mathrm{KHz}$;

c. Spread code length: 31;

d. Spreading gain: 15;

e. Spreading pattern: $m$ sequence.

After debugging and testing the software, the threshold level is up to $-6 \mathrm{~dB}$ and the data communication error rate is 10-5. The anti-interference capability increase about 10dB.

Spread Spectrum Communication Scheme Using 400MHz Ultra-short Wave Band. Signal decline in the million tons of locomotive communication when using ultra-short wave communication. Because the train-tail host is hanging in the rear of the train, the antenna is blocked by the train tail and a weak electric field forms in signal transmission, which affects the signal transmission, making the transmit power of the host difficult. So the spread spectrum communication can effectively reduce the information fading caused by multipath interference. The basic parameters of the spread spectrum are:

a. Frequency: $450 \mathrm{MHz}$;

b. Spread spectrum bandwidth: $5 \mathrm{MHz}$;

c. Spread code length: 512;

d. Spreading gain: 27;

e. Spreading pattern: $m$ sequence.

Digitization Scheme of the Baseband Information. Baseband information mainly includes data information, control information and voice information. The data information and the alarm information of various situations is collected by train-tail device. The information quantity of each frame is about 10 bytes, which can be directly spread spectrum modulated and radio frequency modulated. Control information is mainly a variety of operating commands, error correction coding and address code. Because the voice information has a large amount of information, it should be spread spectrum modulated and Radio frequency modulated after A / D conversion, sampling, compression coding, to reduce data rates. All of the information transmits successively through the two bands, and the data transmission time is about 2.6S. After receiving the information, the radio chooses the processing after determining the bit error rate.

Operation Function Setting Program. The information is transfered in the marshalling station with middle frequency band or ultrashort wave band, whatever the communication happens at the end of the train or between the locomotive stations. So it is necessary to set the communication address information. If the communication runs at different channel, the complexity of the equipment and the difficulty of the equipment management increase, regardless of the frequency division, time division, code division and so on. For the actual situation of the current locomotive dispatching operation, it is possible to communicate with the same frequency in the station communication. Since the probability of communication simultaneously is rare, the difference address can be used on the baseband. Therefore, a communication data with 5 bits number is uesed as the address code, whose nameplate way is fixed on the end of the host shell. The tail of the host of the initial power supply is set as the locomotive "main car" number input column, in order to achieve data communication between the group of locomotive radio and the end of the host "one to one" communication. Voice communication will be on the locomotive shell with a 4-bit number of the address code, because the locomotive group is set randomly. That is, the "main car" number will be inputed by the operator, and "slave car 1", "slave car 2", "slave car 3" will be inputed by the host one. In order to achieve voice communication, only the same number of locomotive radio intercom calls can be used, which makes a "one to one" communication relationship. To avoid communication between other groups of locomotives crosstalk between the phenomenon, "slave car 1", "slave car 2" and " slave car 3" locomotive radios and the end of the host formed a "one to one" 
communication system.

\section{Conclusion}

Spread spectrum communication of the tail device was used in the domestic railway communication for the first time. Digital processing is used at the middle frequency, and most of the radio functions is completed by software, therefore the stable operation of the complex software is very important. The Shenshuo Railway Line has used this system for a long time with many locomotives. The communication test of data communication and voice communication has been run across the mountains, tunnels, corners, stations and plains of the running lines. The test shows data and voice communication function is normal without signal relay. Foremore, the system is stable when it runs at the winter and summer. Clear voice with low noise and modulation shows the good communication performance, which has met the desired requirements.

\section{References}

[1] Fan Changxin, etc. Communication principle [M]. Beijing: National Defense Industry Press, 2001.

[2] Su Tao, Lin Lihua, Lu Guangyue, etc.DSP practical technology [M]. Xi'an: University of Electronic Science and Technology Press, 2002.

[3] Liu Guanglei. Application of the spread spectrum communication in the train-tail safety protection device [J]. Railway Transportation and Economy, 2009 (2): 26-28.

[4] Zhang Huike, Xu Songyi, Shi Liu. Application of M-ary orthogonal spread spectrum technique in scattering communication [J]. Radio communication technology, 2015 (2):82 84.

[5] Zuo Wei. Overview of the development and key technologies of HF communication system [J]. Communication technology, 2014(8):847-853.

[6] Xin Dengsong. Shortwave communication technology and communication frequency selective [J]. Chinese summarized new communication, 2016(4):33-34.

[7] Jiang Hongkui, Liu Min, Kim. A new scheme of 8PSK shortwave communication technology of $[\mathrm{J}]$. Electronic technology, 2016(8):84-86.

[8] Han Xiaoming. Design of emergency communication system based on HF communication technology [J]. Chinese new communications, 2016(8):11-14.

[9] Wang Jinlong. Research and practice of HF Digital Communication [M]. Beijing: Science Press, 2013.

[10]Jiang Bin, Xu Jianliang. A zero intermediate frequency direct sequence spread spectrum digital receiver technology [J]. Electronic design engineering, 2015 (16): 68-70. 